\title{
Corvomeyenia epilithosa sp. nov. (Porifera, Metaniidae) no Parque Nacional da Serra Geral, Rio Grande do Sul, Brasil
}

\author{
Cecilia Volkmer-Ribeiro ${ }^{1,2}$, Rosaria De Rosa-Barbosa ${ }^{1,3}$ \& Vanessa de S. Machado ${ }^{1,4}$ \\ ${ }^{1}$ Museu de Ciências Naturais, Fundação Zoobotânica do Rio Grande do Sul. Caixa Postal 1188, 90001-970 Porto Alegre, \\ Rio Grande do Sul, Brasil. E-mail: cvolkmer@fzb.rs.gov.br \\ ${ }^{2}$ Bolsista Pesquisadora do CNPq. \\ ${ }^{3}$ In memorian. \\ 4 Bolsista BIC-FAPERGS nessabiol@hotmail.com
}

\begin{abstract}
Corvomeyenia epilithosa sp. nov. (Porifera, Metaniidae) at the National Park of Serra Geral, Rio Grande do Sul, Brazil. Corvomeyenia epilithosa sp. nov. is described and registered for a protected area at southern Brazil. An updating of ManconI \& Pronzato's (2002) definition and distribution of genus Corvomeyenia Weltner, 1913 is presented altogether with a taxonomic key for the four species in the genus: $C$. everetti (Mills, 1884) and $C$. carolinensis Harrison (1971), from the Neartic Region, C. thumi (Traxler, 1895) and C. epilithosa sp. nov. from the Neotropical Region. Remarkable habitat distinctions exhibited by these species are commented.

KEY WORDS. Demospongiae, Freswhater sponges, Neartic-Neotropical Regions.
\end{abstract}

RESUMO. Corvomeyenia epilithosa sp. nov. é descrita e registrada para área preservada no Sul do Brasil. São atualizadas a definição e a distribuição do gênero Corvomeyenia Weltner, 1913 propostas por MANCONI \& PrONZATO (2002). É apresentada chave taxonômica para as quatro espécies do gênero: $C$. everetti (Mills, 1884) e C. carolinensis Harrison (1971), para a Região Neártica, C. thumi (Traxler, 1895) e C. epilithosa sp. nov. para a Região Neotropical. São comentadas distinções significativas de habitat dessas espécies.

PALAVRAS CHAVE. Demospongiae, esponjas de água doce, Regiões Neártica-Neotropical.

O Parque Nacional da Serra Geral, Rio Grande do Sul, situa-se na ecorregião da Floresta de Araucária e Campos Sulinos de Altitude (Mма 2000). Justamente nessa região ocorre espécie de esponja considerada endêmica dessa fauna no Brasil: Oncosclera jewelli (Volkmer, 1963), restrita à sub-bacia do Rio Tainhas (Bacia do Rio das Antas). A essa soma-se agora nova espécie do gênero Corvomeyenia Weltner, 1913, ocorrente no rio Tigre Negro, pequena nascente do rio Araranguá, Santa Catarina, situada dentro do Parque acima referido. As duas espécies encontram-se assim em áreas protegidas, a primeira no Parque Estadual do Tainhas e a segunda no Parque Nacional da Serra Geral. Oncosclera jewelli integra, tanto a lista das espécies da fauna ameaçada do Rio Grande do Sul, (decreto 41.672 de 11 de junho de 2002), (FonTANa et al. 2003), quanto a lista nacional da fauna ameaçada (Brasil 2004). O gênero Corvomeyenia, de distribuição NeárticaNeotropical, foi redefinido (VOLKMER-RIBEIRo 1992), contendo duas espécies da primeira região, C. everetti (Mills, 1884) e C. carolinensis Harrison, 1971, e apenas uma espécie da segunda, C. thumi (Traxler, 1895), com distribuição restrita ao Brasil e, nesse, ao bioma Cerrado, de Roraima até São Paulo. Devido ao fato de Corvomeyenia epilithosa sp. nov. completar o par Neotropical de espécies do gênero e, ainda, de representar distribuição em área de clima temperado frio, sendo única espécie do gênero ocorrendo em ambiente lótico, com características taxonômicas distintas das demais, seu registro e descrição revestem-se de um significado especial em termos do contexto ecológico, biogeográfico e sistemático que representam.

O trabalho oportuniza ainda retificar o texto de MANCONI \& Pronzato (2002), quando C. thumi foi omitida, tanto do gênero, quanto da obra, sem qualquer justificativa, com a conseqüente distribuição do gênero restrita à Região Neártica e ao conhecimento disponível em 1971, data da descrição de C. carolinensis.

\section{MATERIAL E MÉTODOS}

Os materiais foram coligidos manualmente nos exercício de 1993 e 1994, no Rio Tigre Preto, Parque Nacional da Serra Geral (2904'48,77"S, 4959'18,65"W) (Figs 1 e 2), conservados a seco e depositados na coleção de Porifera do Museu de Ciências Naturais da Fundação Zoobotânica do Rio Grande do Sul (MCN-FZB). Foram medidas 50 espículas de cada categoria (megascleras, microscleras e gemoscleras) do holótipo (MCNPOR 2844) e dos parátipos (MCN-POR 2793, 2784, 2803, 2799). As preparações das espículas para exames ao microscópio óptico 
e ao microscópio eletrônico de varredura (MEV) seguiram Volkmer-Ribeiro \& Turce (1996). A terminologia utilizada na caracterização das espículas segue BouRy-EsNaUlt \& RÜTZLER (1997). As fotos ao MEV foram realizadas no Centro de Microscopia Eletrônica da Pontifícia Universidade Católica do Rio Grande do Sul (PUCRS), Porto Alegre. A comparação com Corvomeyenia everetii foi feita sobre o exemplar MCN-POR 1229, doado pelo Dr. Thomas M. Frost e coligido pelo mesmo em Little Rock Lake, Wisconsin, USA, em 26.IX.1984; com Corvomeyenia carolinensis foi feita sobre o exemplar MCN-POR 1156, que constitui preparação de fragmento do holótipo, doado pelo Dr. K. Rützler, Smithsonian Institution, Washington, D.C. USA, e coligido por F.W. Harrison em Adams Pond, Richmond County, South Carolina, USA, em 28/I/1967; e com Corvomeyenia thumi foi feita sobre os exemplares MCN-POR 1431, coletado pelo Dr. Pedro S. Mera, em 23.IX.1987, no Rio Uraricoera, Ilha de Maracá, Alto Alegre, Roraima, Brasil; MCN-POR 1510, 1515, 2316, coletados por C. Volkmer-Ribeiro, em 4.XII.1987, na mesma localidade e MCN-POR 1935, coletado pelo Dr. Pedro S. Mera, em 13.VII.1985, à 7 Km da BR 163, Oriximiná, Pará, Brasil.

\section{RESULTADOS}

\section{Metaniidae Volkmer-Ribeiro, 1986 Corvomeyenia Weltner, 1913}

Corvomeyenia Weltner, 1913; Harrison, 1971; Volkmer-Ribeiro, 1992; Manconi \& Pronzato, 2002.

Diagnose. Esponjas com uma ou duas categorias de gemoscleras birrotuladas, diferindo em tamanho, com eixos longos, lisos, delicados, rótulas desiguais, recortadas em um número irregular de ganchos ou dentes, microscleras compondo uma ou duas categorias, no primeiro caso microbirrotuladas retas, com eixo liso ou espinhoso, com rótulas regularmente recortadas, no segundo fortemente curvas e com rótulas reduzidas, assumindo o aspecto de chelas.

\section{Corvomeyenia epilithosa sp. nov.}

\section{Figs 1-21, Tab. I}

Diagnose. Gemoscleras birrotuladas, constituindo duas categorias distintas em tamanho, eixos robustos, lisos, retos ou fortemente encurvados, às vezes com expansões medianas, rótulas fortemente umbonadas, dissimilares em tamanho e recortadas em número variável de ganchos, esses com disposições irregulares. As gemoscleras apresentam numerosas alterações de curvatura. Microscleras constituindo apenas uma categoria: birrotuladas diminutas, de tamanhos bastante variáveis, com eixos retos ou levemente curvos, robustos, lisos ou espinhosos e rótulas umbonadas, recortadas em ganchos. Espécie de Corvomeyenia de ambiente lótico (Fig. 1).

Descrição. Esponjas aderidas a substratos rochosos, formando crostas verdes, descontínuas, extremamente delgadas, de consistência delicada e superfície lobulada (Fig. 2). Esquele- to da placa basal sem organização definida das espículas, passando a colunar e a seguir a reticulado, em direção à superfície (Fig. 5). Fibras esqueletais poliespiculares, com espongina abundante na porção mediana do esqueleto, superfície da esponja híspida, devido à projeção das fibras da camada superior. Pinacoderme espesso, incrustado com microscleras (Figs 4 e 8). Ósculos pequenos, agrupados em áreas osculares (Fig. 8). Gêmulas pequenas, arredondadas, contidas em locus esféricos do esqueleto (Fig. 3).

Megascleras óxeas curtas, lisas e levemente curvas, com eventuais expansões medianas, extremidades gradualmente aguçadas (Figs 6 e 18, Tab. I).

Gemoscleras como na diagnose da espécie (Figs 7 e 18, Tab. I).

Microscleras birrotuladas extremamente abundantes, de uma única categoria, porém com variações de tamanho e curvatura dos eixos, diminutas, com eixos retos ou levemente curvos, robustos, lisos ou espinhados e rótulas recortadas em números irregulares de ganchos (Figs 8, 9 e 18, Tab. I).

Gêmulas raras, pequenas, esféricas, contidas em locus esféricos do esqueleto (Fig. 3); camada pneumática delgada, provida de espongina alveolar. Camada gemular externa fina com incrustação de megascleras. As gemoscleras ultrapassam a camada pneumática e ajudam a sustentar a camada externa, de modo a formarem grandes espaços aéreos entre uma e outra (Figs 10 e 13). Tubo foraminal alongado, projetado na superfície da gêmula e provido de colarinho de espongina (Fig. 13).

Etimologia. Do grego, epi = sobre e lithos = pedra, uma vez que essa é a única espécie do gênero ocorrente em ambiente lótico e em substrato rochoso.

Habitat. Ambiente lótico, raso, torrentoso, desenvolvido em fundo rochoso basáltico, com águas cor de café oriundas da drenagem de turfeiras de altitude, portanto ácidas (Fig. 1).

Distribuição. A espécie foi detectada, até o presente, somente na localidade tipo, situada nas proximidades da vila de Cambará, Cambará do Sul, RS. O rio corre no sentido leste-oeste, indo precipitar-se no "canyon" da Fortaleza (Fig. 20), integrando assim a bacia do rio Araranguá, no Estado de Santa Catarina. O local de ocorrência encontra-se dentro dos limites do Parque Nacional da Serra Geral, e, no sítio de ocorrência, o rio tem seu curso paralelo à estrada de rodagem que, penetrando no Parque, leva até a borda do "canyon". Considerando-se a cobertura dos levantamentos dessa fauna já operados pela autora e colaboradores, particularmente no região do Planalto (DE RosABARBOSA 1984), a nova espécie apresenta-se, em caráter preliminar, como endêmica do ecossistema de Floresta com Araucária.

Localidade Tipo. Rio Tigre Preto, Parque Nacional da Serra Geral, 2904'48,77"S, 4959'18,65"W, Cambará do Sul, Rio Grande do Sul, Brasil (Figs 20 e 21).

Holótipo. Brasil, Rio Grande do Sul: Cambará do Sul (Parque Nacional da Serra Geral, 2904'48,77"S, 4959'18,65"W, Rio Tigre Preto), 06.IV.1994, C.V. Ribeiro leg. (MCN 2844). Depositado na coleção de Porifera do Museu de Ciências Naturais 

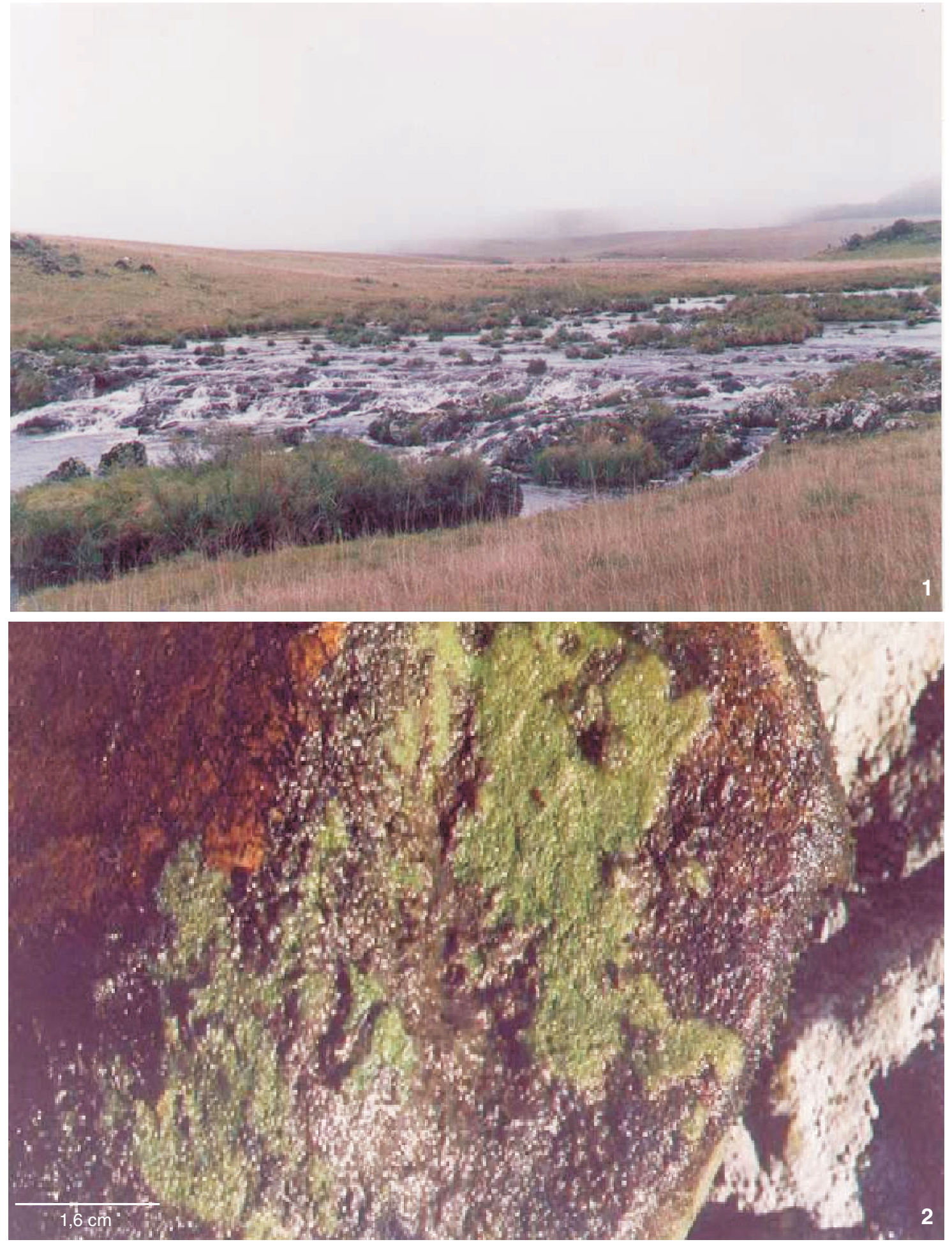

Figuras 1-2. (1) Rio Tigre Preto, localidade-tipo de Corvomeyenia epilithosa sp. nov., fotografado no outono, Parque Nacional da Serra Geral, Cambará do Sul, RS, Brasil. Foto: C. Volkmer-Ribeiro; (2) espécime vivo de Corvomeyenia epilithosa sp. nov. fotografado na localidade-tipo. Foto: Ingrid Heydrich.

Revista Brasileira de Zoologia 22 (4): 844-852, dezembro 2005 


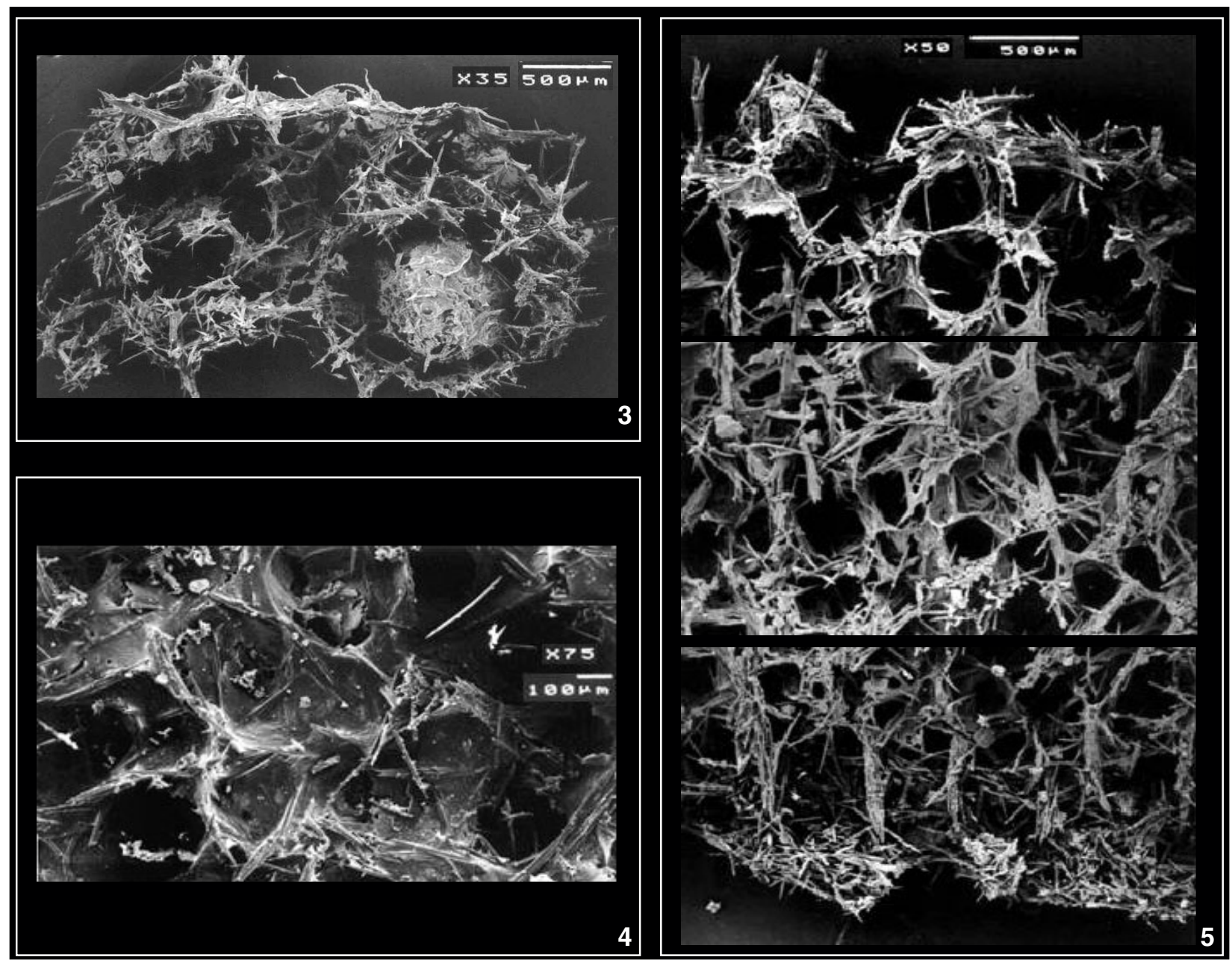

Figuras 3-5. Fotos ao MEV de estruturas de Corvomeyenia epilithosa sp. nov.: (3) situação da gêmula no esqueleto; (4) pinacoderme com incrustação de microscleras; (5) estrutura diferenciada do esqueleto da base ao pinacoderme.

Tabela I. Medidas ( $\mu \mathrm{m})$, das dimensões das espículas de C. epilithosa sp. nov.

\begin{tabular}{|c|c|c|c|c|c|c|c|c|}
\hline & \multicolumn{4}{|c|}{ Comprimento } & \multicolumn{4}{|c|}{ Largura } \\
\hline & Mínima & Máxima & Média & Desvio Padrão & Mínima & Máxima & Média & Desvio Padrão \\
\hline Megasclera & 211,0 & 457,0 & 290,2 & 44,26 & 3,7 & 29,6 & 16,9 & 6,90 \\
\hline Gemosclera & 60,3 & 127,2 & 102,1 & 16,32 & 1,6 & 8,4 & 5,5 & 1,16 \\
\hline Microsclera & 16,7 & 39,2 & 26,7 & 5,95 & 1,7 & 7,4 & 2,9 & 0,77 \\
\hline
\end{tabular}

da Fundação Zoobotânica do Rio Grande do Sul (MCN-FZB). Parátipos. BRASIL, Rio Grande do Sul: Cambará do Sul (Parque Nacional da Serra Geral, 2904'48,77"S, 4959'18,65"W, Rio Tigre Preto), 04.XI.1993 (MCN-POR 2784); 12.I.1994 (MCN-POR 2799, 2803, 2805, 2806, 2807); 12.I.1994 (2793, 2802); 12.I.1994 (MCN-POR 2804); 06.IV.1994 (MCN-POR 2843); 07.VI.1994 (MCN-POR 2858); 02.VI.2000 (MCN-POR 4572, 4573), C. V. Ribeiro leg.
Outros materiais examinados. Corvomeyenia everetti: MCNPOR 1229, Thomas M. Frost leg. Little Rock Lake, Wisconsin, USA, 26.IX.1984. Corvomeyenia carolinensis: MCN-POR 1156, (esquizolótipo) F.W. Harrison leg. Adams Pond, Richmong County, S. Carolina, USA, 28/I/1967. Corvomeyenia thumi: MCN-POR 1431, Pedro S. Mera leg., Rio Uraricoera, Ilha de Maracá, Alto Alegre, Roraima, Brasil, 23.IX.1987; MCN-POR 1510, 1515, 2316, C. Volkmer-Ribeiro leg., Rio Uraricoera, Ilha de Maracá, Alto Alegre, 

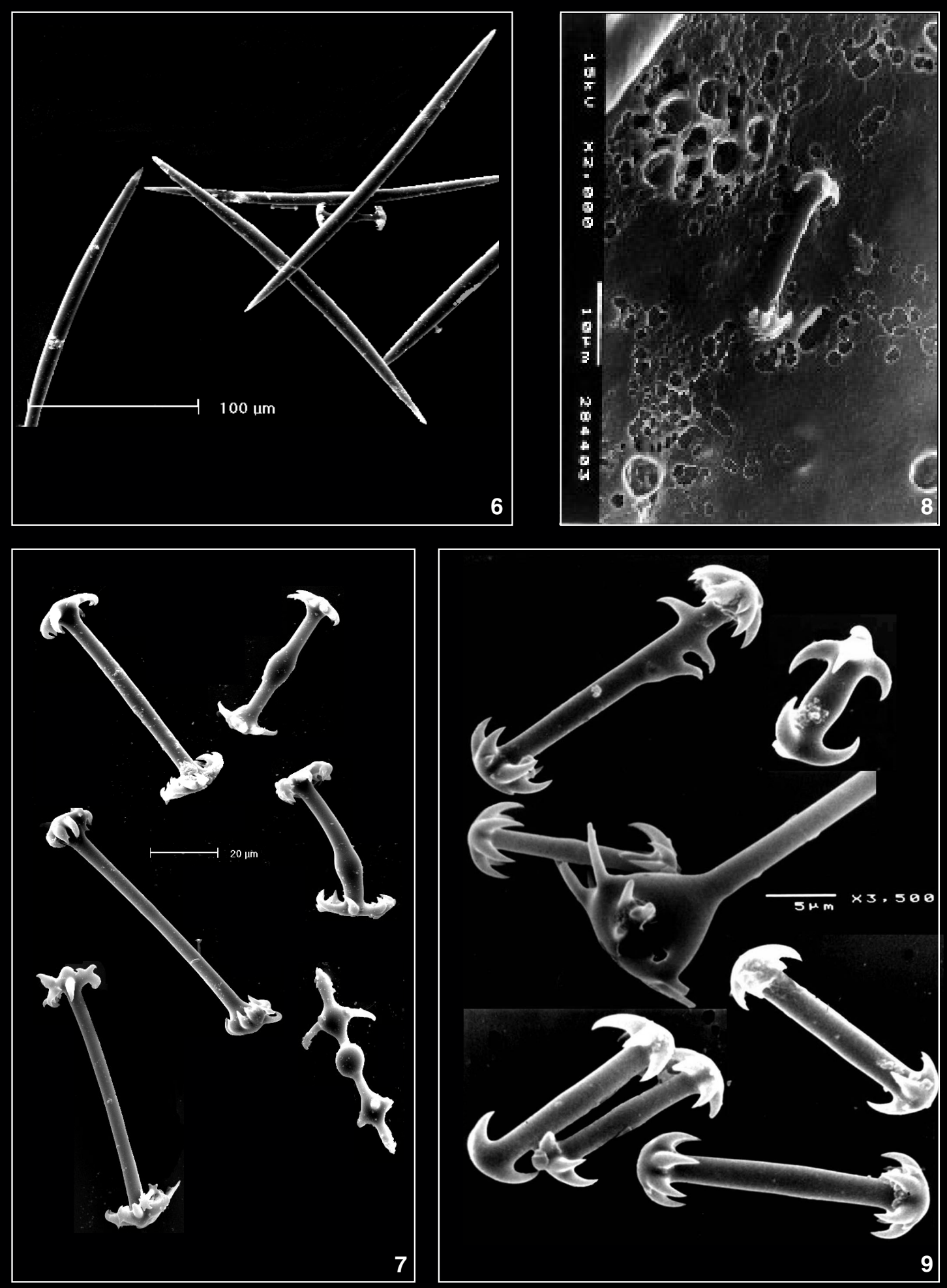

Figuras 6-9. Fotos ao MEV das espículas de Corvomeyenia epilithosa sp. nov.: (6) megascleras; (7) gemoscleras; (8) microsclera in situ ao lado de área oscular; (9) conjunto de microscleras e detalhe da rótula de gemosclera em formação.

Revista Brasileira de Zoologia 22 (4): 844-852, dezembro 2005 

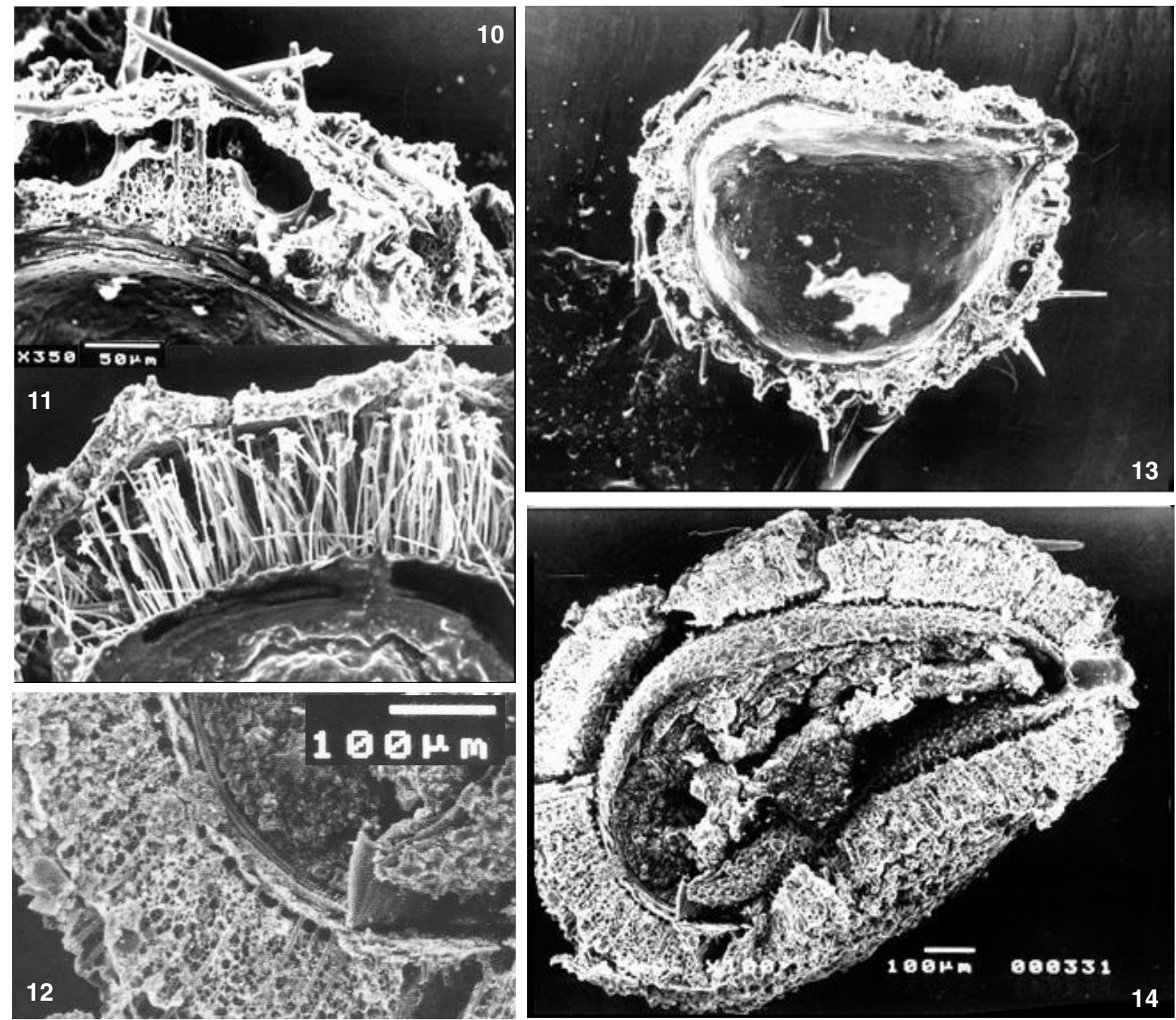

Figuras 10-14. Fotos ao MEV de secções transversais da parede das gêmulas de: (10) Corvomeyenia epilithosa sp. nov.; (11) C. everetti; (12) C. thumi; (13) C. epilithosa sp. nov.; (14) C. thumi; possibilitando uma comparação da diferença de tamanho e estrutura das gêmulas nas espécies neotropicais do gênero.

Roraima, Brasil, 4.XII.1987; MCN-POR 1935, Pedro S. Mera leg., à 7 Km da BR 163, Oriximiná, Pará, Brasil, 13.VII.1985.

\section{Chave para as espécies de Corvomeyenia}

1. Gêmulas com somente uma categoria de gemoscleras: birrotuladas boletifomes, com os ganchos marginais da rótula inferior soldados de modo a compor uma rótula expandida, achatada, enquanto os ganchos marginais da rótula superior, menor, em forma de calombo, permanecem livres (Figs 12, 14 e 15) ..................................... C. thumi (Traxler, 1895)

1'. Gêmulas com duas categorias de gemoscleras, diferindo em tamanho
2. Duas categorias de microscleras presentes: chelas e birrotuladas (Fig. 16) ...................... C. carolinensis Harrison, 1971

2'. Microscleras somente da categoria birrotulada ................ 3

3. A camada pneumática das gêmulas apresenta-se drasticamente reduzida ou ausente (Figs 11 e 17) .....

C. everetti (Mills, 1884)

3'. A camada pneumática das gêmulas apresenta-se dividida em duas porções: uma interna constituída por espaços aéreos regulares e pequenos; e outra mais externa, constituída por algumas grandes vesículas aéreas (Figs 10 e 13)

C. epilithosa sp. nov. 


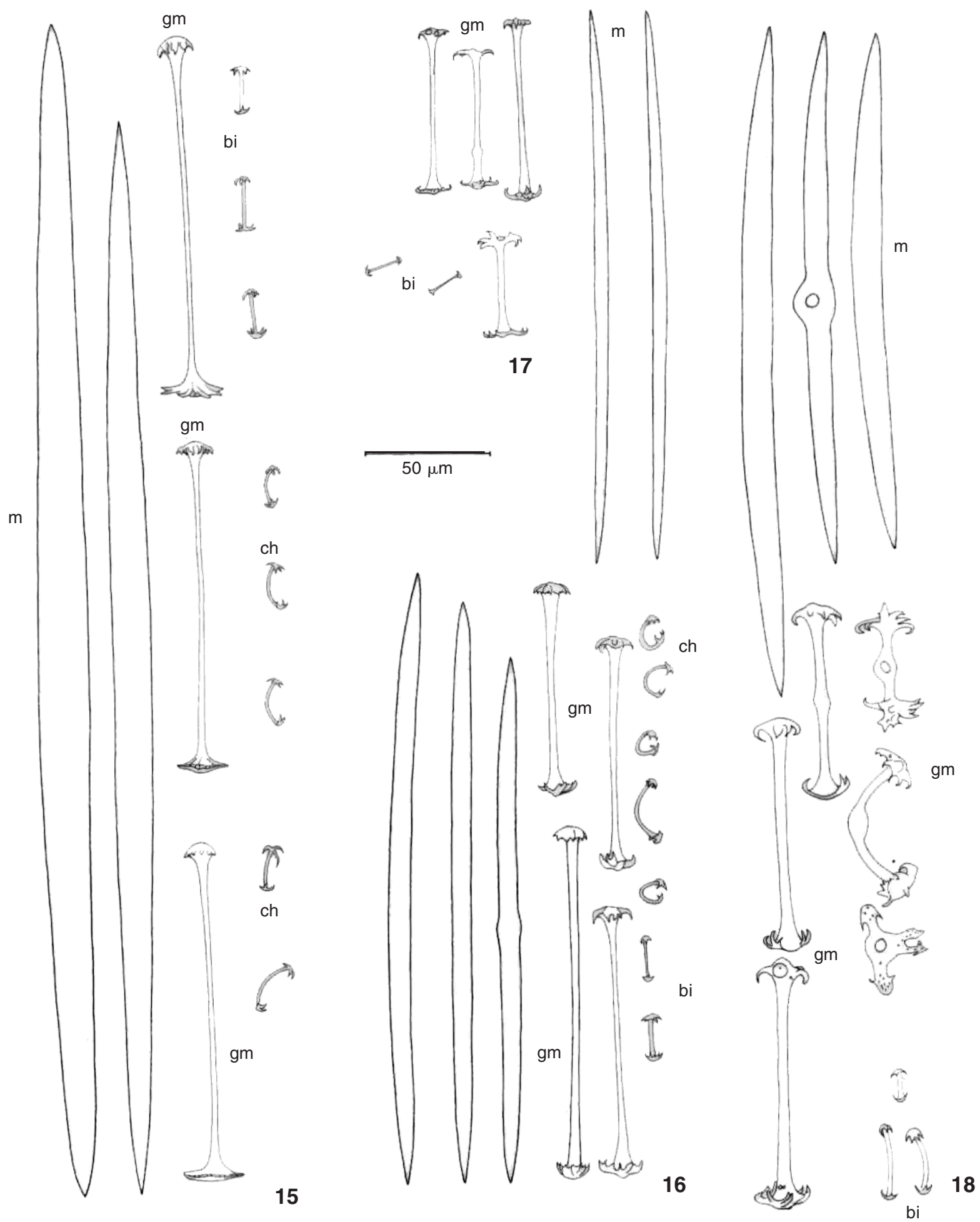

Figuras 15-18. Desenhos em câmera clara dos conjuntos espiculares das quatro espécies do gênero Corvomeyenia 15. C. thumi; 16. C. carolinensis; 17. C. everetti; 18. C. epilithosa sp. nov. 


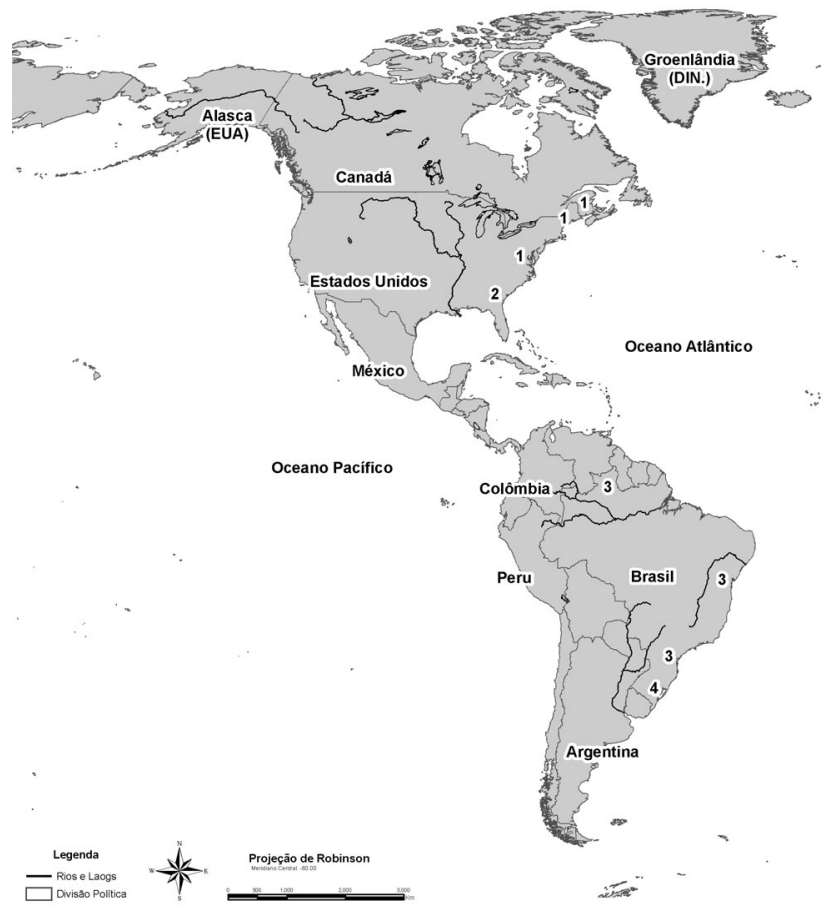

Figura 19. Mapa das Regiões Neártica e Neotropical indicando as áreas de distribuição das quatro espécies do gênero Corvomeyenia: 1) C. everetti, 2) C. carolinensis, 3) C. thumi, 4) C. epilithosa sp. nov.

\section{DISCUSSÃO}

A nova espécie distingue-se das demais do gênero, tanto pela estrutura das gêmulas, quanto pelas características das espículas e do habitat. É a única espécie de Corvomyenia que apresenta a camada pneumática com grandes espaços aéreos situados fora da espongina alveolar e a única a ocorrer em ambiente lótico, e além disso, turbulento (Fig. 1). Destaca-se assim o valor seletivo das câmaras aéreas extras em suas gêmulas, facilitando a dispersão por flutuação. C. epilithosa sp. nov. distingue-se ainda por constituir crostas perenes, já que as restantes espécies de Corvomeyenia são sazonais. No entanto essa esponja tem em comum com suas congêneres a preferência por águas de turfeiras, com pH ácido e ricas em substâncias húmicas, ocupando substratos colocados praticamente na interface águaar, portanto com abundância de oxigênio. A esponja viva tem cor verde esmeralda devido à associação com zoochlorelas, outra característica do gênero e que se alia ao potencial para plena exposição solar, essa decorrente da ocorrência em superfície.

O gênero apresenta-se agora constituído por dois pares de espécies, um par na Região Neártica, e outro par da região Neotropical (Fig. 19). O primeiro com C. everetti, com preferência por clima temperado e C. carolinensis, em clima subtropical. O segundo com o par da América do Sul, com a nova espécie situada em região de clima temperado e C. thumi característica

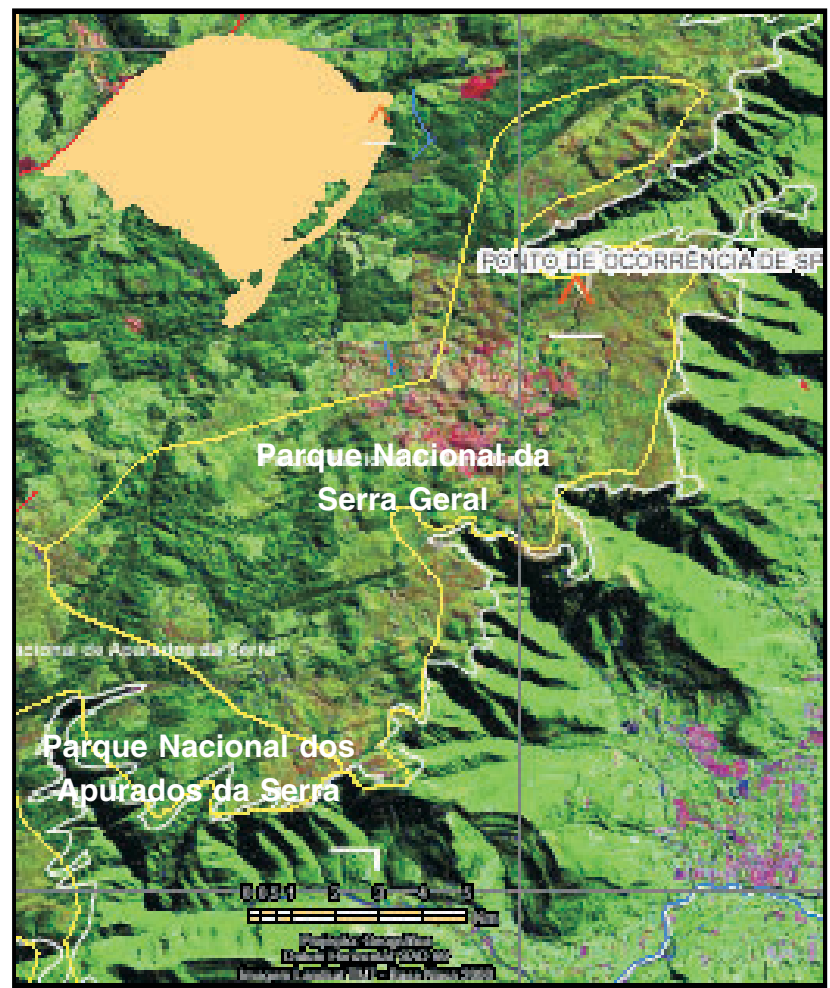

Figura 20. Imagem de satélite, mostrando a delimitação do Parque Nacional da Serra Geral e indicando o local de ocorrência de C. epilithosa sp. nov.

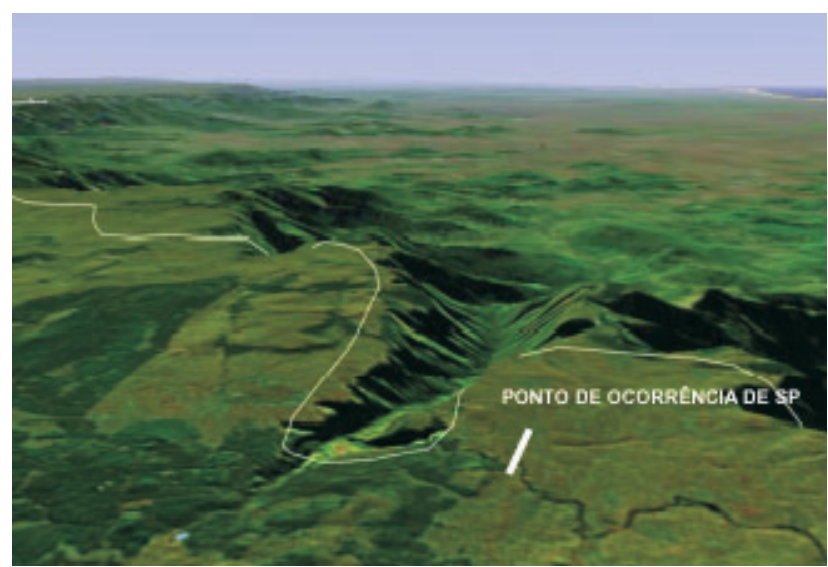

Figura 21. Imagem de satélite, mostrando o curso do Rio Tigre Preto em direção ao canyon Fortaleza, com indicação do local de ocorrência de C. epilithosa sp. nov.

de áreas de cerrado tropicais à subtropicais.

O gênero Corvomeyenia foi incluído na familia Metaniidae (VolKmer-Ribeiro 1986) dado seu conjunto espicular complexo, considerado pela autora com afinidades filogenéticas com

Revista Brasileira de Zoologia 22 (4): 844-852, dezembro 2005 
Metania Gray (1867), em que a rótula menor das gemoscleras mostra tendência para redução a um calombo quase desprovido de ganchos, tendência essa acentuada em C. carolinensis e, sobretudo, em $C$. thumi, espécie sul-americana, redescrita após a descoberta de espécimes vivos (Volkmer-Ribeiro 1992). Manconi \& Pronzato (2002) aceitam a proposta dessa inclusão a nível de família, mas omitem a existência da espécie neotropical, com o que o gênero é apresentado como restrito à região Neártica. O presente registro de espécie de Corvomeyenia em clima temperado da região Neotropical, obrigou a redefinição do gênero, dadas as características marcantes da nova espécie. A ampliação da distribuição de Corvomeyenia ilustra o potencial desse gênero para distribuir-se nas duas faixas climáticas temperadas do globo.

Devido ao fato de C. epilithosa sp. nov. completar o par Neotropical de espécies do gênero e, ainda, de representar a distribuição em área austral de clima temperado (Cambará é um município que tem invernos com neve e congelamento de espelhos d'água e de água encanada), sendo a única espécie do gênero ocorrendo em ambiente lótico, sua preservação reveste-se de um significado muito importante em termos do contexto ecológico, biogeográfico e filogenético que aporta.

Dado o registro de endemismo que, no presente se configura, aliado ao pequeno contingente populacional existente na localidade tipo, planteia-se uma situação até certo ponto digna de cuidados especiais, para a conservação dessa espécie. Qualquer alteração das condições físicas e/ou químicas do pequeno manancial onde foi encontrada, como por exemplo, alterações no solo da bacia de captação, por mudança da cobertura vegetal ou por denudação ou, ainda, execução de barramentos de qualquer tipo, alterando de modo permanente a altura da coluna d'água, poderão levar ao desaparecimento de C. epilithosa sp. nov. no local.

O fato da localidade tipo estar contida dentro de Parque Nacional (Fig. 20) e de confirmar-se, em data recente, a continuidade da existência da esponja no local, indica uma garantia de resguardo do habitat. No entanto, preocupa a acessibilidade do manancial, extremamente próximo à estrada de rodagem, que leva à borda do "canyon" (Fig. 21) e, por isto mesmo, de acesso fácil para turistas. Dada a pequena magnitude do manancial e seu curso em áreas de pretérita pastagem extensiva, planteiam-se como ameaças à espécie projetos de manejo do Parque, outros que os até aqui praticados.

\section{AGRADECIMENTOS}

As autoras agradecem ao Biólogo Paulo César Reis Bastos, chefe do Parque Nacional da Serra Geral, IBAMA, pelos dados locais fornecidos, bem como pela autorização para o desenvolvimento do projeto; aos Dr. Thomas M. Frost ("in memoriam") pela doação de fragmento de Corvomeyenia everetti e ao Dr. Klaus Rützler, USNM, Smithsonian Institution, pela doação de preparação de fragmento do holótipo de Corvomeyenia carolinensis; à pesquisadora Ingrid Heydrich, à desenhista Rejane Rosa e a Arlete Pasqualetto, do Laboratório de Geoprocessamento, do Museu de Ciências Naturais da Fundação Zoobotânica do Rio Grande do Sul por, respectivamente, a foto da figura 2 , a arte final dos desenhos nas figuras 15-18, ao mapa da figura 19 e às imagens de satélite das figuras 20 e 21; aos consultores da Revista Brasileira de Zoologia, Dr. Eduardo C.M. Hajdu, Museu Nacional, UFRJ, e anônimo, pelas valiosas sugestões apresentadas.

\section{REFERÊNCIAS BIBLIOGRÁFICAS}

Boury-Esnault, N. \& Rützler, K. 1997. Thesaurus of Sponge Morphology. Smithsonian Contributions to Zoology, Washington, 596: 1-55.

BRASIL. 2004 . Instrução Normativa nº 5, de 21 de maio de 2004. Diário Oficial da República Federativa do Brasil, Brasília, 28 de maio de 2004, Seção 1, p.136-142.

De Rosa-Barbosa, R. 1984. Reavaliação da fauna espongológica continental do Estado do Rio Grande do Sul, Brasil, frente a novas coletas. Iheringia, Série Zoologia, Porto Alegre, 64: 127-148.

Fontana, C.S.; G.A. Bencke \& R.E. ReIs. 2003. Livro vermelho da fauna ameaçada de extinção no Rio Grande do Sul. Porto Alegre, Edipucrs, 632p.

HarRison, F.W. 1971. A taxonomical investigation of the Genus Corvomeyenia Weltner (Spongillidae) with an introduction of Corvomeyenia carolinensis sp. nov. Hydrobiologia, The Hague, 38 (1): 123-140.

Manconi, M. \& R. Pronzato. 2002. Surb-order Spongillina subord. nov.: freshwater sponges, p. 921-1019. In: J.N.A. HOOPER \& R.W.M. van SOEST (Eds). Systema Porifera: A guide to the Classification of Sponges. New York, Kluwer Academic, Plenum Publ., vol. 1, 1101p.

MMA. 2000. Avaliação e ações prioritárias para a conservação da biodiversidade da Mata Atlântica e Campos Sulinos. Brasília, Ministério do Meio Ambiente, SBF, 40p.

VolKmer-Ribeiro, C. 1986 Evolutionary study of the freswhater sponge genus Metania Gray, 1867: III. Metaniidae, new family. Amazoniana, Kiel, 9 (4): 493-509.

Volkmer-Ribeiro, C. 1992. The freshwater sponges in some peatbog ponds in Brazil. Amazoniana, Kiel, 12 (2): 317-335.

Volkmer-Ribeiro, C. \& B. J. Turce, 1996. SEM analysis of silicious spicules of a freshwater sponge indicate paleoenvironmental changes. Acta Microscopica, Rio de Janeiro, 5 (6): 186-187.

Weltner, W. 1913. Süsswasserschwämme (Spongillidae) der Deutschen Zentralafrika. Expedition 1907-1908. Ergebn. Deutsch. Zentralafr. Exped. (Zoologie), Berlin, 4: 475-485.

Recebido em 11.III.2005; aceito em 26.IX.2005. 